\title{
Estructura comunitaria de diatomeas presentes en los sedimentos superficiales de ocho lagos andinos de Chile central
}

\author{
Diatoms community structure in superficial sediments of eight Andean lakes \\ of central Chile
}

\author{
INGRID E. ALVIAL ${ }^{1 *}$, FABIOLA J. CRUCES $^{1}$, ALBERTO E. ARANEDA $^{1}$, MARTIN GROSJEAN $^{2}$ \\ \& ROBERTO E. URRUTIA ${ }^{1}$
}

\footnotetext{
${ }^{1}$ Centro de Ciencias Ambientales EULA, Universidad de Concepción, Barrio Universitario s/n, Casilla 160 C, Concepción, Chile

${ }^{2}$ Institute of Geography/NCCR-Climate, Universität Bern, Hallerstrasse 12 CH-3012, Bern, Switzerland; *e-mail para correspondencia: ialvial@icaro.dic.uchile.cl
}

\begin{abstract}
RESUMEN
En este trabajo se analizó la estructura comunitaria de diatomeas depositadas en los sedimentos superficiales de ocho lagos andinos chilenos ubicados entre los $32^{\circ} 49^{\prime}$ y $38^{\circ} 48^{\prime}$ S. Se encontró un total de 99 taxa de diatomeas, distribuidos en 48 géneros. Del total de taxa, 74 fueron identificados a nivel de especie siendo todos ellos cosmopolitas a excepción de Eunotia andinofrequens, Gomphonema punae, Pinnularia araucanensis y Pinnularia acidicola, entre otras, que están descritos solo para el Hemisferio Sur. Por otro lado, las muestras analizadas presentaron distinta composición florística de diatomeas. De esta manera, en los ensambles diatomológicos depositados en los sedimentos de las lagunas de altura Ocho, Huifa, Ensueño y Negra ubicadas sobre los $2.860 \mathrm{~m}$ del nivel del mar, abundaron especies bentónicas, típicas de aguas oligotróficas y ácidas como Achnanthidium exiguum, Achnanthidium minutissimum, Encyonema minutum, Pinnularia acidicola y Planothidium lanceolatum. En los ensambles diatomológicos de los lagos Galletué, Icalma y Laja, ubicados bajo los $1.360 \mathrm{~m}$ del nivel del mar, abundaron diatomeas planctónicas, características de aguas alcalinas y mesotróficas como Asterionella formosa, Aulacoseira distans, Aulacoseira granulata, Cyclotella stelligera y Rhopalodia gibba.
\end{abstract}

Palabras clave: composición de especies, Bacillariophyceae, sedimento superficial, lagos andinos, Chile.

\begin{abstract}
In this research the taxonomic structure of diatoms in sediments of high mountain lakes was studied. These lakes are located in Chile between $32^{\circ} 49^{\prime}$ and $38^{\circ} 48^{\prime} \mathrm{S}$ in the Andean Cordillera. A total of 99 diatom taxa distributed in 48 genera were identified and all this taxa are cosmopolitan excepting a Eunotia andinofrequens, Gomphonema punae, Pinnularia araucanensis and Pinnularia acidicola, which are know only for the Southern Hemisphere. The assemblages of diatoms were different in the studied lakes. So the high mountain lakes Ocho, Huifa, Ensueño and Negra, dominated benthic diatoms which are typical of oligotrophic and acid waters as Achnanthidium exiguum, Achnanthidium minutissimum, Encyonema minutum, Pinnularia acidicola and Planothidium lanceolatum. In the assemblages from lakes Galletué, Icalma and Laja planktonic diatoms were more abundant, which are common in alkaline and mesotrophic waters, e.g., Asterionella formosa, Aulacoseira distans, Aulacoseira granulata, Cyclotella stelligera and Rhopalodia gibba.
\end{abstract}

Key words: species composition, Bacillariophyceae, superficial sediment, Andean lake, Chile.

\section{INTRODUCCIÓN}

Las diatomeas (Bacillariophyceae) constituyen un componente importante en las comunidades del fitoplancton, pero gran parte de sus especies son sésiles y están asociadas a comunidades bentónicas y litorales (Round et al. 1990). No obstante, la mayoría de los trabajos ecológicos y biogeográficos de estas microalgas se efectúan sobre la base de las comunidades que habitan en el fitoplancton.

Por otro lado, en los sedimentos lacustres es posible encontrar tanto formas bentónicas como formas provenientes de la columna de agua 
(Reynolds et al. 1982) por esta razón, los sedimentos entregan un registro temporal y espacial de lo que habita en el sistema lacustre (Anderson \& Battarbee 1994).

En razón de lo anterior, las diatomeas depositadas en los sedimentos lacustres pueden entregar información ambiental de los procesos transcurridos en el ecosistema acuático (Brugam 1983). Esto es posible, porque entre las capas sedimentarias que se depositan lentamente y con poca perturbación se van archivando los restos silíceos de las diatomeas que pueden ser utilizadas como indicadores de las condiciones ambientales del lago al momento de depositarse (Smol \& Cumming 2000). Como señalan Charles et al. (1994), muchas perturbaciones antrópicas y naturales que han afectado a los lagos pueden ser evaluadas mediante la utilización de los restos de diatomeas almacenados en los sedimentos. La utilización de estas microalgas en este tipo de estudio incluye la inferencia de cambios ambientales pasados tales como eutrofización (Christie \& Smol 1993), acidificación (Flower 1986), salinidad (Gell 1998), climáticos (Bigler \& Hall 2002) y procesos naturales como erupciones volcánicas (Cruces et al. 2006).

Una de las dificultades para la realización de estudios basados en diatomeas es la falta de información acerca de la composición y distribución de las especies en una región geográfica de interés (Smol \& Cumming 2000).

En relación a los trabajos de diatomeas realizados en nuestro país es preciso mencionar que el conocimiento de la flora diatomológica de aguas continentales es escaso, limitándose a determinadas zonas geográficas del país, a saber, zona Norte y zona Centro-sur (Urrutia et al. 2000, Cruces et al. 2001, Cruces et al. 2006, Rivera 2006).

Un área geográfica importante para el estudio de las diatomeas es la cordillera de los Andes. $\mathrm{Su}$ gran extensión longitudinal que atraviesa zonas climáticas muy diferentes y su desarrollo paralelo a la costa crean las condiciones idóneas para que se desarrollen especies endémicas (Rumrich et al. 2000). Este dominio geográfico se encuentra poco estudiado siendo posible mencionar en los Andes del norte de Chile los trabajos de Tapia et al. (2004), Díaz \& Maidana (2005) y Rivera \& Cruces (2005); en los Andes de Chile central, los estudios de Rivera (1970), Parra et al. (1993) y Cruces et al. (2006) y en toda su extensión norte-sur, el trabajo de Rumrich et al. (2000).

En la actualidad un proyecto en desarrollo se encuentra realizando estudios en un grupo de lagos cordilleranos del centro-norte de Chile de los cuales cinco están contemplados en el presente estudio. Estos sistemas están lejos de ser intervenidos por actividades humanas dado su difícil acceso lo que mantiene sus condiciones prístinas y los convierten en unidades de estudio interesantes de conocer la diversidad y composición florística de diatomeas. La composición de ensambles bentónicos de estos cinco sistemas fue comparada a la flora bentónica de tres sistemas cordilleranos ubicados en el centro-sur de Chile. De acuerdo a lo señalado, el objetivo de este trabajo fue estudiar y comparar la composición florística y diversidad de ensambles de diatomeas presentes en los sedimentos superficiales de ocho cuerpos lacustres ubicados en los Andes del centronorte y centro-sur de Chile. Este trabajo constituye una primera aproximación al conocimiento de la flora de diatomeas que habita en lagos altoandinos chilenos.

\section{MATERIALES Y MÉTODOS}

\section{Área de estudio}

El área de estudio abarcó dos sectores de la cordillera de los Andes: el primero ubicado entre los $32^{\circ} 49^{\prime} \mathrm{S}$ y $34^{\circ} 06^{\prime} \mathrm{S}$ y el segundo entre los $37^{\circ} 26^{\prime} \mathrm{S}$ y $38^{\circ} 48^{\prime} \mathrm{S}$ (Fig. 1). El primer sector incluyó a las lagunas del Inca, Ocho, Ensueño, Huifa y Negra, ubicadas sobre los $2.860 \mathrm{~m}$ del nivel del mar. El clima en esta parte de los Andes es de tipo templado frío de altura, con abundantes precipitaciones de nieve $(0,6$ a 6,4 m) y lluvias $(28,5$ a $471,7 \mathrm{~mm})$ en los meses de invierno (junio-septiembre) y una estación estival seca (diciembre-marzo) que alcanza los $16{ }^{\circ} \mathrm{C}$ (CONAF 2004). Vila \& Mühlhauser (1987) mencionan que en los lagos de altura el régimen térmico depende de la latitud y altitud, siendo la mayoría de ellos polimícticos, con frecuentes periodos de mezcla total; o amícticos, permanentemente estratificados. Los datos limnológicos publicados en la Tabla 1 fueron tomados por Grosjean et al. (resultados no publicados) entre los meses de noviembre y 
marzo de los años 2004 y 2005. Los contenidos de nutrientes medidos en el periodo de muestreo revelan una condición de oligotrofia para estos lagos. El pH, conductividad y temperatura en estas lagunas fueron medidos directamente por un conductivímetro marca Hanna y los datos de los principales iones fueron analizados en el Laboratorio de Química Ambiental del Centro de Ciencias Ambientales EULA de la Universidad de Concepción.

El segundo sector en estudio incluyó a los lagos Laja, Icalma y Galletué, situados en la precordillera de los Andes, bajo los $1.360 \mathrm{~m}$ de altitud y en las zonas de nacimiento del sistema fluvial del río Biobío (Parra et al. 1993). De acuerdo a este autor, los lagos Galletué e Icalma están sometidos a fuertes variaciones climáticas entre invierno y verano, presentando estratificación en verano y marcada circulación en invierno, lo que los clasifica como lagos monomícticos temperados. Además, este autor clasifica a estos lagos de acuerdo a sus observaciones como oligotróficos. El clima de este sector es templado húmedo con abundantes precipitaciones entre los meses de mayo a agosto, también acompañadas de nevadas. Las temperaturas más bajas alcanzan los $-0,3{ }^{\circ} \mathrm{C}$ en invierno y $18{ }^{\circ} \mathrm{C}$ en verano (CONAF 2004).

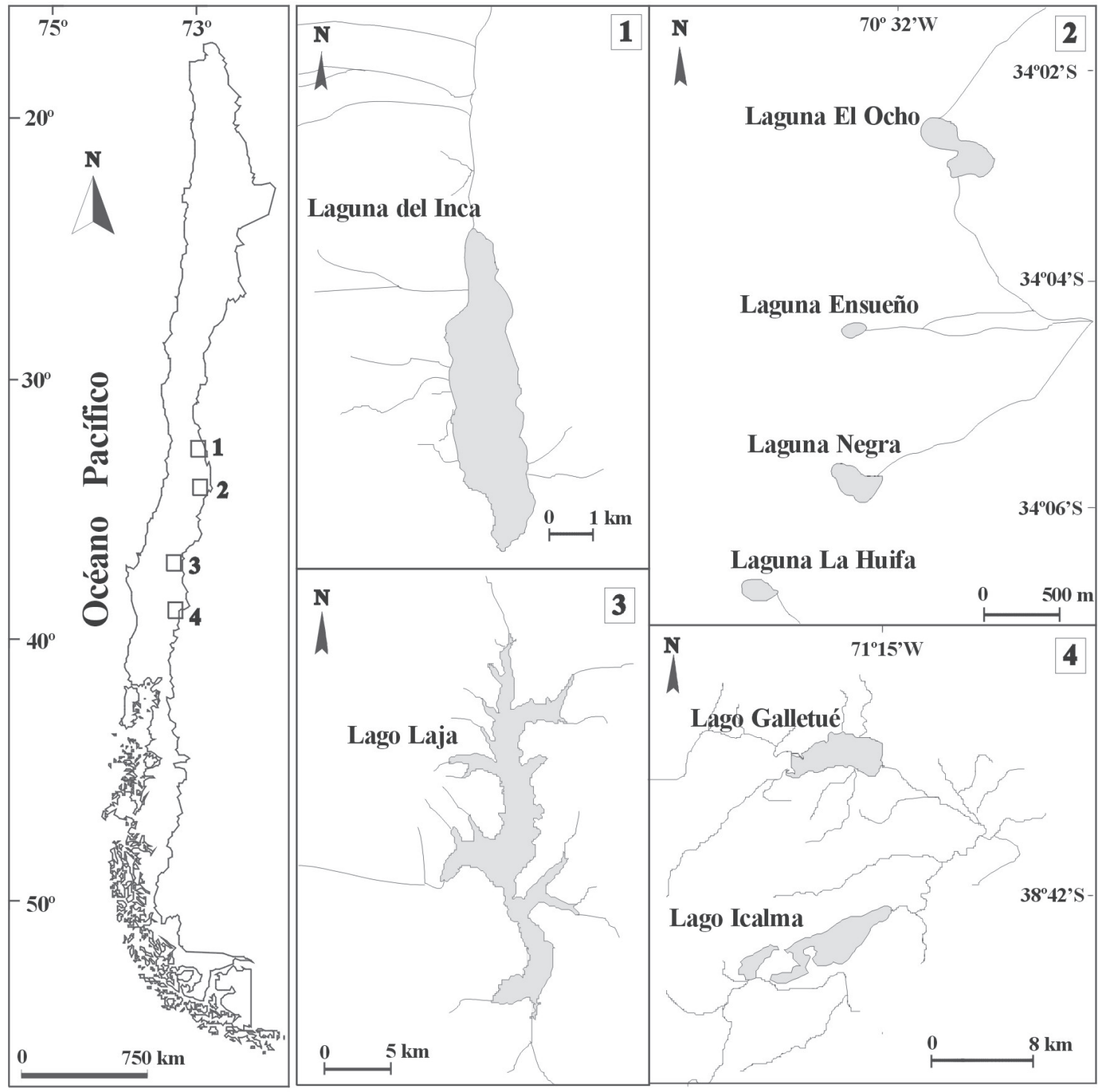

Fig. 1: Ubicación de los lagos estudiados en la cordillera de los Andes Location of the study lakes in the Andean Cordillera. 
La caracterización ambiental y limnológica de los datos de calidad de agua de los lagos Icalma y Galletué se obtuvo del trabajo realizado por Parra et al. (1993). Por su parte la información limnológica del lago Laja fue obtenida por Urrutia et al. (resultados no publicados) en febrero del año 1999. Los datos de los lagos Icalma y Galletué presentados en la Tabla 1 corresponden al promedio de valores medidos entre los meses de enero y marzo del año 1991.

\section{Preparación y análisis de las muestras de diatomeas}

Se analizaron los ensambles de diatomeas contenidas en los sedimentos superficiales de columnas sedimentarias recolectas en la parte más profunda de cada lago. Para ello, se utilizó un "gravity corer" marca Uwitec. Las columnas sedimentarias de las lagunas del Inca, Huifa, Ocho, Negra y Ensueño fueron obtenidas entre los meses de noviembre y marzo de los años 2004 y 2005. Por su parte las columnas sedimentarias pertenecientes a los lagos Laja, Icalma y Galletué fueron obtenidas entre los meses de enero y febrero del año 2000.
De cada una de las muestras se tomó $0,1 \mathrm{~g}$ de sedimento seco, el cual fue oxidado con peróxido de hidrógeno y/o ácido sulfúrico, siguiendo las metodologías convencionales de Hasle \& Fryxell (1970) y Battarbee (1986). Posteriormente se realizaron preparaciones permanentes con resina Hyrax (IR $=1,7)$, las que fueron analizadas cualitativamente para objetivos taxonómicos y cuantitativamente para determinar las abundancias relativas de las especies, contando un mínimo de 500 valvas de diatomeas por cada preparado en duplicado. La identificación de los frústulos de diatomeas se realizó utilizando un microscopio fotónico Carl Zeiss con aumento de 1000x y un microscopio electrónico de barrido JEOL/DEO y esta identificación siguió a Krammer \& LangeBertalot (1991, 2000), Barber \& Carter (1996) y Rumrich et al. (2000).

\section{Tratamiento de datos}

Se determinó la diversidad específica mediante el calculo del índice de diversidad de ShannonWiener $\left(\mathrm{H}^{\prime}\right)$, la riqueza $\left(\mathrm{H}_{\text {máx }}=\log _{2} \mathrm{~S}\right.$, donde $\mathrm{S}$ es el número de taxa) y la equidad $\left(\mathrm{H}^{`} / \mathrm{H}_{\text {máx }}\right)$ en las asociaciones de diatomeas (Krebs 1989).

TABLA 1

Localización geográfica y algunos parámetros limnológicos medidos en los lagos estudiados

Geographic location and some limnological parameters measured in the Andean lakes

\begin{tabular}{|c|c|c|c|c|c|c|c|c|}
\hline Parámetro & $\begin{array}{l}\text { Laguna } \\
\text { del Inca }\end{array}$ & $\begin{array}{c}\text { Laguna } \\
\text { Ocho }\end{array}$ & $\begin{array}{l}\text { Laguna } \\
\text { Ensueño }\end{array}$ & $\begin{array}{c}\text { Laguna } \\
\text { Huifa }\end{array}$ & $\begin{array}{c}\text { Laguna } \\
\text { Negra }\end{array}$ & $\begin{array}{l}\text { Lago } \\
\text { Laja }\end{array}$ & $\begin{array}{c}\text { Lago } \\
\text { Galletué }\end{array}$ & $\begin{array}{l}\text { Lago } \\
\text { Icalma }\end{array}$ \\
\hline Altitud (m) & 2.850 & 3.620 & 3.510 & 3.482 & 3.528 & 1.360 & 1.150 & 1.152 \\
\hline Profundidad máxima (m) & 73,3 & 43,7 & 4,5 & 3,8 & 9,9 & 135 & 45 & 35 \\
\hline Latitud (S) & $32^{\circ} 49^{\prime}$ & $34^{\circ} 02^{\prime}$ & $34^{\circ} 04^{\prime}$ & $34^{\circ} 06^{\prime}$ & $34^{\circ} 06^{\prime}$ & $37^{\circ} 26^{\prime}$ & $38^{\circ} 40^{\prime}$ & $38^{\circ} 48^{\prime}$ \\
\hline Longitud (W) & $70^{\circ} 07^{\prime}$ & $70^{\circ} 32^{\prime}$ & $70^{\circ} 33^{\prime}$ & $70^{\circ} 32^{\prime}$ & $70^{\circ} 32^{\prime}$ & $71^{\circ} 15^{\prime}$ & $71^{\circ} 15^{\prime}$ & $71^{\circ} 15^{\prime}$ \\
\hline $\mathrm{pH}$ & 8,5 & 6,6 & 6,6 & 6,9 & 4,3 & 7,2 & 7,0 & 7,4 \\
\hline Conductividad $\left(\mu \mathrm{S} \mathrm{cm}^{-1}\right)$ & 108 & 71,9 & 88,3 & 143,8 & 127,4 & 25 & 51,6 & 47,7 \\
\hline Temperatura $\left({ }^{\circ} \mathrm{C}\right)$ & 12,2 & 10,7 & 14,0 & 11,7 & 13,3 & 12 & 13,7 & 13,6 \\
\hline $\mathrm{SO}_{4}\left(\mathrm{mg} \mathrm{g}^{-1}\right)$ & 18,6 & 28,5 & 40,7 & 69,9 & 59,2 & 5 & 1,6 & 6,8 \\
\hline $\mathrm{NO}_{3}\left(\mathrm{mg} \mathrm{g}^{-1}\right)$ & 0,43 & 0,001 & 0,03 & 0,04 & 0,009 & 0,005 & 0,005 & 0,025 \\
\hline $\mathrm{Na}\left(\mathrm{mg} \mathrm{g}^{-1}\right)$ & 4,2 & 1,7 & 2,6 & 4,3 & 4,7 & 1,8 & 2,6 & 1,8 \\
\hline $\mathrm{Mg}\left(\mathrm{mg} \mathrm{g}^{-1}\right)$ & 1,9 & 0,5 & 1,56 & 1,2 & 1,4 & 1,1 & 1,3 & 1 \\
\hline $\mathrm{Ca}\left(\mathrm{mg} \mathrm{g}^{-1}\right)$ & 19,7 & 14,5 & 13,6 & 28,6 & 16 & 3,2 & 4,8 & 4,4 \\
\hline $\mathrm{K}\left(\mathrm{mg} \mathrm{g}^{-1}\right)$ & 0,4 & 0,2 & 0,3 & 0,2 & 0,7 & 0,6 & 0,5 & 0,5 \\
\hline
\end{tabular}


Mediante un protocolo de "bootstrap" se calcularon intervalos de confianza del $95 \%$ para comparar los valores del índice de diversidad de Shannon-Wiener en las muestras (Hammer et al. 2001).

Se generaron dendrogramas utilizando el método de agrupamiento por promedio aritmético no ponderado (UPGMA, por sus siglas en inglés). Debido a que las muestras presentaron un alto número de especies dominantes y raras, la similitud fue medida por el índice de Morisita (Wolda 1981). Para esto, se utilizaron los datos de abundancia relativa transformados a $\log \left(\log _{10}\right)$. La robustez de los dendrogramas se determinó por valores de "bootstrap" (Manly 1997). Todos los cálculos fueron realizados en el programa PASTPalaeontological Statistics, ver. 1.50 (Hammer et al. 2001).

\section{RESULTADOS}

\section{Composición taxonómica}

En los estratos superficiales de los ocho lagos estudiados se encontró un total de 99 taxa de diatomeas distribuidos en 48 géneros (Tabla 2). Del total de taxa, 74 fueron identificados a nivel de especie, siendo todos ellos cosmopolitas a excepción de Eunotia andinofrequens (Lange-Bertalot) LangeBertalot, Gomphonema araucana LangeBertalot, Pinnularia araucanensis LangeBertalot, Rumrich \& Krammer y Pinnularia acidicola Van der Vijver \& Le Cohu, que están descritos solo para el Hemisferio Sur. De las especies identificadas, solamente un $12 \%$ presentó abundancias mayores al $10 \%$ en al menos una de las muestras y fueron definidas como "dominantes", un $45 \%$ presentó abundancias entre 1 y $10 \%$ y el resto presentó abundancias menores al $1 \%$ y se las catalogó como "raras".

Los géneros con mayor número de especies fueron Eunotia (10 especies), Pinnularia (siete especies), Nitzschia (siete especies) Fragilaria (seis especies), Navicula (seis especies) y Gomphonema (cinco especies). En laguna Negra, las especies más abundantes fueron Pinnularia acidicola Van der Vijver \& Le Cohu $(53,2 \%)$ y Aulacoseira alpigena (Grunow) Krammer (14\%), ambas especies acidófilas (Catalán et al. 1993, Van der Vijver 2004). En laguna Ensueño dominaron las especies cosmopolitas, bentónicas y alcalófilas Encyonema minutum (Hilse) D. G. Mann (19,3 \%) y Planothidium lanceolatum (Brébisson) Lange-Bertalot $(17,6 \%)$. En laguna Huifa dominó Eolimna $s p(77 \%)$. En laguna del Inca las especies más abundantes fueron Discostella stelligera Cleve \& Grunow $(54,4 \%)$ y Fragilaria $c f$. mazamaensis (Sovereign) Lange-Bertalot $(40,5 \%)$. D. stelligera es cosmopolita, planctónica y habita en un amplio rango de condiciones tróficas (Wunsam et al. 1995). Por su parte, F. cf. mazamaensis es una especie cosmopolita y de mayor abundancia en aguas alcalinas (Wunsam et al. 1995). En laguna Ocho dominaron $F$. cf. mazamaensis $(18,6 \%), P$. lanceolatum $(16,6 \%)$ y Achnanthidium exiguum Krasske (16,3\%). En lago Laja abundaron especies cosmopolitas y planctónicas como Asterionella formosa Hassall (14,6 \%) y Aulacoseira distans (Ehrenberg) Simonsen $(27,9 \%)$. En lago Galletué dominó Aulacoseira granulata (Ehrenberg) Simonsen $(55,6 \%)$. Finalmente en lago Icalma dominó D. stelligera (49 \%) y Staurosirella pinnata (Ehrenberg) Willians \& Round (13\%).

\section{Diversidad de especies}

El número de especies $(S)$ en los ensambles diatomológicos analizados fue mayor en la laguna Ensueño (41) y menor en la laguna Huifa (12) (Tabla 3).

La diversidad específica medida a través del índice de Shannon-Wienner presentó los valores más altos en laguna Ensueño $(3,3)$ y laguna Ocho $(2,9)$, mientras que los valores más bajos fueron encontrados en laguna del Inca $(1,3)$ y laguna Huifa $(1,5)$. La equidad fluctuó entre 0,73 en la laguna Ocho y 0,27 en la laguna del Inca. A pesar de los resultados anteriores, el intervalo de confianza del $95 \%$ mostró que la diversidad, la riqueza y la equidad no mostraron diferencias significativas entre los ensambles de diatomeas depositados en los sedimentos de los lagos de altura y en lagos andinos del sur de Chile. Sin embargo, los ensambles desde ambos grupos de lagos fueron caracterizados por presentar pocas especies en altas abundancias. 
TABLA 2

Lista de especies de diatomeas encontradas en los sedimentos de los lagos andinos estudiados que presentaron abundancia relativa $>3 \%$ en al menos una muestra

Checklist of identified diatoms species of the Andean lakes studied with relative abundance $>3 \%$ at least in one sample

\begin{tabular}{|c|c|c|c|c|c|c|c|c|c|c|}
\hline Taxa & $\begin{array}{c}\text { Categoría } \\
\text { ID }\end{array}$ & Hábitat & $\begin{array}{l}\text { Laguna } \\
\text { Ensueño }\end{array}$ & $\begin{array}{l}\text { Laguna } \\
\text { Huifa }\end{array}$ & $\begin{array}{l}\text { Laguna } \\
\text { Negra }\end{array}$ & $\begin{array}{l}\text { Laguna } \\
\text { del Inca }\end{array}$ & $\begin{array}{l}\text { Laguna } \\
\text { Ocho }\end{array}$ & $\begin{array}{l}\text { Lago } \\
\text { Laja }\end{array}$ & $\begin{array}{c}\text { Lago } \\
\text { Galletué }\end{array}$ & $\begin{array}{l}\text { Lago } \\
\text { Icalma }\end{array}$ \\
\hline Achnanthidium exiguum Krasske & 1 & $\mathrm{P}, \mathrm{N}, 0, \mathrm{M}$ & 0,4 & - & - & - & 16,3 & - & - & - \\
\hline A. minutissimum (kützing) Czarnecki & 1 & $\mathrm{~B}, \mathrm{~N}, \mathrm{O}, \mathrm{M}$ & 3,3 & 5,1 & - & - & 5,0 & - & - & 1,4 \\
\hline Asterionella formosa Hassall & 1 & $\mathrm{~B}, \mathrm{~N}, \mathrm{M}$ & - & - & - & - & - & 14,6 & 6,9 & 7,2 \\
\hline Aulacoseira alpigena (Grunow) Krammer & 1 & $\mathrm{P}, \mathrm{A}, 0$ & - & - & 14,0 & - & 6,4 & - & - & - \\
\hline A. distans (Ehrenberg) Simonsen & 1 & $\mathrm{P}, \mathrm{A}, 0$ & - & - & - & - & - & 27,9 & - & 0,72 \\
\hline A. granulata (Ehrenberg) Simonsen & 1 & $\mathrm{P}, \mathrm{N}, 0, \mathrm{M}$ & - & - & - & - & - & 3,8 & 55,6 & - \\
\hline Bacillaria sp. & 4 & - & 0,4 & - & - & 0,36 & 1,9 & 0,2 & - & 1,8 \\
\hline Brachysira cf. brebisonii Ross & 3 & - & 0,2 & - & - & - & - & - & - & - \\
\hline Cyclotella cf. glomerata Bachmann & 3 & - & - & - & 0,6 & - & - & 6,3 & - & - \\
\hline C. meneghiniana Kützing & 1 & $\mathrm{P}, \mathrm{N}, \mathrm{M}$ & - & - & - & - & - & 0,1 & 3,1 & - \\
\hline C. stelligera (Cleve \& Grunow) Van Heurck & 1 & $\mathrm{P}, \mathrm{N}, 0, \mathrm{M}$ & - & - & - & 54,4 & - & 7,7 & 2,8 & 49 \\
\hline Encyonema minutum (Hilse) D. G. Mann & 1 & - & 19,3 & 0,76 & - & 0,7 & - & - & - & 1,1 \\
\hline Eolimna sp. & 4 & - & 5,0 & 77 & - & 0,9 & 7,1 & - & - & 1,6 \\
\hline Eunotia sp.1 & 4 & - & - & - & 4,7 & - & - & - & - & - \\
\hline Eunotia sp.2 & 4 & - & - & - & 3,2 & - & - & - & - & - \\
\hline E. paludosa Grunow & 1 & $\mathrm{~B}, \mathrm{~A}, 0$ & - & - & 3,1 & - & - & - & - & - \\
\hline E. pectinalis (Dillwyn) Rabenhorst & 1 & $\mathrm{~B}, \mathrm{~A}, \mathrm{M}$ & 3,3 & - & - & - & - & 0,2 & - & - \\
\hline E. diodon Ehrenberg sensu lato & 1 & $\mathrm{~B}, \mathrm{~A}, 0, \mathrm{M}$ & - & - & 4,9 & - & - & - & - & - \\
\hline E. exigua (Brébisson) Rabenhorst & 1 & $\mathrm{~B}, \mathrm{~A}, 0$ & - & - & 5,7 & - & - & - & - & - \\
\hline Fragilaria capucina Desmazieres & 1 & $\mathrm{~B}, \mathrm{~N}, \mathrm{M}$ & 3,5 & - & - & 0,2 & - & 2,3 & - & \\
\hline F.cf. mazanaensis (Sovereign) Lange-Bertalot & 1 & - & 2,8 & - & - & 40,5 & 18,6 & - & - & - \\
\hline Frustulia saxónica Rabenhorst & 1 & $\mathrm{P}, \mathrm{A}, 0$ & - & - & 6,2 & - & - & - & - & - \\
\hline G. punae Lange-Bertalot & 2 & - & 1,5 & 2,1 & - & - & 3,1 & - & - & - \\
\hline G. parvulum Kützing & 1 & $\mathrm{P}, \mathrm{N}, \mathrm{M}$ & 3,0 & - & - & - & - & - & - & - \\
\hline Hannaea arcus (Ehrenberg) Patrick & 1 & - & - & - & - & - & - & 3,4 & 0,4 & - \\
\hline Navicula sp. & 4 & - & 4,2 & - & - & 0,36 & - & - & - & 0,54 \\
\hline N. iridis (Ehrenberg) Cleve & 1 & $\mathrm{P}, \mathrm{N}, \mathrm{O}$ & 0,6 & 0,76 & 3,6 & - & - & - & - & - \\
\hline N. amphibia Grunow & 1 & $\mathrm{P}, \mathrm{N}, \mathrm{M}$ & - & - & - & - & - & 4,9 & 0,6 & 0,18 \\
\hline N. cf. perminuta (Grunow) M. Peragallo & 3 & $P, N, 0$ & 1,3 & - & - & - & 3,8 & - & - & - \\
\hline P. acidicola Van de Vijver \& Le Cohu & 2 & $\mathrm{P}, \mathrm{A}, 0$ & - & - & 53,2 & - & 4,8 & - & - & - \\
\hline \multicolumn{11}{|l|}{ Planothidium lanceolatum (Brébisson) } \\
\hline Lange-Bertalot & 1 & $\mathrm{P}, \mathrm{N}, \mathrm{M}$ & 17,6 & 9,0 & 1,2 & 0,54 & 16,6 & 0,2 & 0,2 & 0,72 \\
\hline Rhopalodia gibba (Ehrenberg) 0. Muller & 1 & $\mathrm{P}, \mathrm{N}, \mathrm{M}$ & - & - & - & - & 0,3 & 4,5 & 1,1 & 0,54 \\
\hline Sellaphora pupula (Kützing) Mereschkowsky & 1 & & 6,5 & - & - & - & - & - & - & 0,36 \\
\hline S. construens Ehrenberg & 1 & $\mathrm{P}, \mathrm{N}, \mathrm{M}$ & 4,4 & - & - & - & 1,9 & 4,9 & 7,9 & 8,8 \\
\hline Staurosirella pinnata Ehrenberg & 1 & $\mathrm{P}, \mathrm{N}, \mathrm{M}$ & 3,6 & - & - & - & - & 3,5 & - & 13,0 \\
\hline Ulnaria ulna (Kützing) Compère & 1 & $\mathrm{P}, \mathrm{N}, \mathrm{M}$ & 0,2 & - & - & 0,54 & 1,4 & 3,9 & 2,4 & 3,4 \\
\hline \multicolumn{11}{|l|}{ Urosolenia eriensis (H.L. Sm.) } \\
\hline Round \& R. M. Crawford & 1 & - & - & - & - & - & - & - & 4,7 & 0,18 \\
\hline
\end{tabular}

Identificación (ID): (1) Especie cosmopolita conocida; (2) Especie conocida en el hemisferio sur; (3) Taxa cercano a una especie conocida; (4) Especie indeterminada de un género conocido (Rumrich et al. 2000). Hábitat: P (planctónica), B (bentónica); A (acidófila), N (neutrófila y/o alcalófila), O (oligotrófica), M (mesotrófica), - (sin información) 


\section{TABLA 3}

Riqueza específica, diversidad y equidad en los ensambles diatomológicos

Species richness, diversity and eveness in the diatomologic assemblages

\begin{tabular}{lccc}
\hline Sistema lacustre & Riqueza específica $(S)$ & Índice de diversidad de Shannon-Wienner $\left(\mathrm{H}^{\prime}\right)$ & Equidad $\left(\mathrm{H}^{\prime} / \operatorname{lnS}\right)$ \\
\hline Laguna Ensueño & 41 & 3,3 & 0,6 \\
Laguna Huifa & 12 & 1,5 & 0,4 \\
Laguna del Inca & 13 & 1,3 & 0,3 \\
Laguna Negra & 20 & 2,2 & 0,4 \\
Laguna Ocho & 25 & 2,9 & 0,7 \\
Lago Laja & 33 & 2,8 & 0,5 \\
Lago Galletué & 24 & 1,9 & 0,3 \\
Lago Icalma & 31 & 2,6 & 0,4 \\
\hline
\end{tabular}

Patrones de similitud en la composición de especies

De acuerdo al análisis de cluster, las asociaciones de diatomeas depositadas en los estratos superficiales fueron agrupadas en tres grupos (Fig. 2). El primer grupo separó a laguna Negra que presentó una composición de especies dominada por taxa tolerantes a condiciones ácidas como $P$. acidicola, A. alpigena y varias especies del género Eunotia. El segundo grupo incluyó a los lagos Laja, Icalma y Galletué, donde dominaron especies planctónicas como $A$. granulata, A. formosa, Rhopalodia gibba (Ehrenberg) O Müll, Nitzschia amphibia Grunow, Staurosira construens (Ehrenberg) Grunow y Cyclotella meneghiniana Kützing. Finalmente el tercer grupo abarcó a las lagunas de altura Ensueño, del Inca, Ocho y Huifa, en las cuales las especies más características fueron $P$. lanceolatum, E. minutum, Gomphonema punae Lange-Bertalot, Gomphonema gracile Ehrenberg, Eolimna sp., A. minutisimum y Neidium iridis (Ehrenberg) Cleve.

Los valores de bootstrap para este cluster indican una buena robustez del dendrograma (valores sobre el $50 \%$ ) validando la existencia de los tres grupos antes mencionados.

\section{DISCUSIÓN}

El presente trabajo es el primero en estudiar y comparar la composición de ensambles de diatomeas en sistemas lacustres de altura en la cordillera de los Andes.
Los resultados obtenidos indican que la flora diatomológica estudiada desde los sedimentos superficiales en los ocho sistemas lacustres andinos presenta una elevada cantidad de especies cosmopolitas o de amplia distribución geográfica.

Considerando la información bibliográfica disponible, de las 99 especies encontradas, 67 son cosmopolitas y las restantes incluyen a especies descritas solo en el hemisferio sur y taxa que no fue posible su identificación a nivel específico. Es importante mencionar que la mayor proporción de especies cosmopolitas fue encontrada en los lagos Laja, Icalma y Galletué ubicados en la parte centro-sur de nuestro país, mientras que la mayor cantidad de especies indeterminadas (Fig. 4) se encontró en las lagunas de altura Ocho, Ensueño, Huifa y Negra ubicadas por sobre los $3.000 \mathrm{~m}$ del nivel del mar en la parte centro del país.

Los valores del índice de diversidad de Shannon-Wiener fluctuaron entre 3,3 (laguna Ensueño) y 1,3 (laguna del Inca). No obstante, al compararlas mediante el protocolo de "bootstrap" (95 \% de confianza), se observó que los ensambles de diatomeas no mostraron diferencias significativas en los valores de diversidad. A partir esto, es posible inferir que las lagunas de altura Ensueño, Ocho, Negra y Huifa no siguen necesariamente el patrón general observado en lagos ubicados en condiciones climáticas extremas, como es presentar baja riqueza y diversidad de especies (Rautio et al. 2000, Kilroy et al. 2006). Lo anterior se sustenta en el hecho de que la flora de lagos de altura presenta interesantes 
adaptaciones al medio como resultado de las peculiares características de estos lagos: intensa irradiación solar, frecuentes periodos de estratificación y mezcla por alta turbulencia y baja cantidad de gases e iones en solución (Vila \& Muhlhauser 1987). Además, las diatomeas es uno de los pocos grupos de algas que pueden vivir en una amplia variedad de hábitats, incluso bajo condiciones extremas, desde hielos polares hasta aguas termales (Round et al. 1990). Sin embargo, los ensambles de diatomeas se caracterizaron por presentar pocas especies en altas abundancias. Esto parece caracterizar a los lagos de altura, donde la disponibilidad de recurso y las condiciones extremas permiten el desarrollo de unas pocas especies dominantes (Rautio et al. 2000).

El análisis de similitud por su parte, ordenó los ensambles de diatomeas encontrados en los sedimentos superficiales de los lagos andinos en tres grupos. De esta manera lagos Icalma, Galletué y Laja presentaron ensambles compuestos por especies cosmopolitas, planctónicas y de condiciones mesotróficas.
Por su parte, en las lagunas del Inca, Ensueño, Huifa y Ocho, se encontraron diatomeas de distribución cosmopolita y diatomeas que podrían presentar una distribución local. Esto se sustenta en el hecho de que varios de estos taxa se encuentran citados por Rumrich et al. (2000) como posibles nuevas especies. Tal es el caso de Eolimna sp. encontrada en sistemas lacustres del Altiplano chileno (Lámina 71 y figura 5-8, página 382 de Runrich et al. 2000 y Fig. 4G del presente trabajo) y que fue altamente abundante en los sedimentos de la laguna Huifa (77 \%). Finalmente el tercer agrupamiento correspondió a la laguna Negra. En este sistema se encontraron diatomeas típicas de aguas con valores bajos de $\mathrm{pH}$ como $P$. acidicola (Fig. 3K y 3L), A. alpígena (Fig. $3 \mathrm{~A}$ y $3 \mathrm{~B})$ y varias especies del género Eunotia (Fig. 4F, 4J y 4K). En relación a lo anterior, varios autores han señalado la importancia que tendría el pH de las aguas en determinar la distribución y composición de las especies de diatomeas en los sistemas naturales (Van Dam et al. 1998, DeNicola 2000). En este sentido,

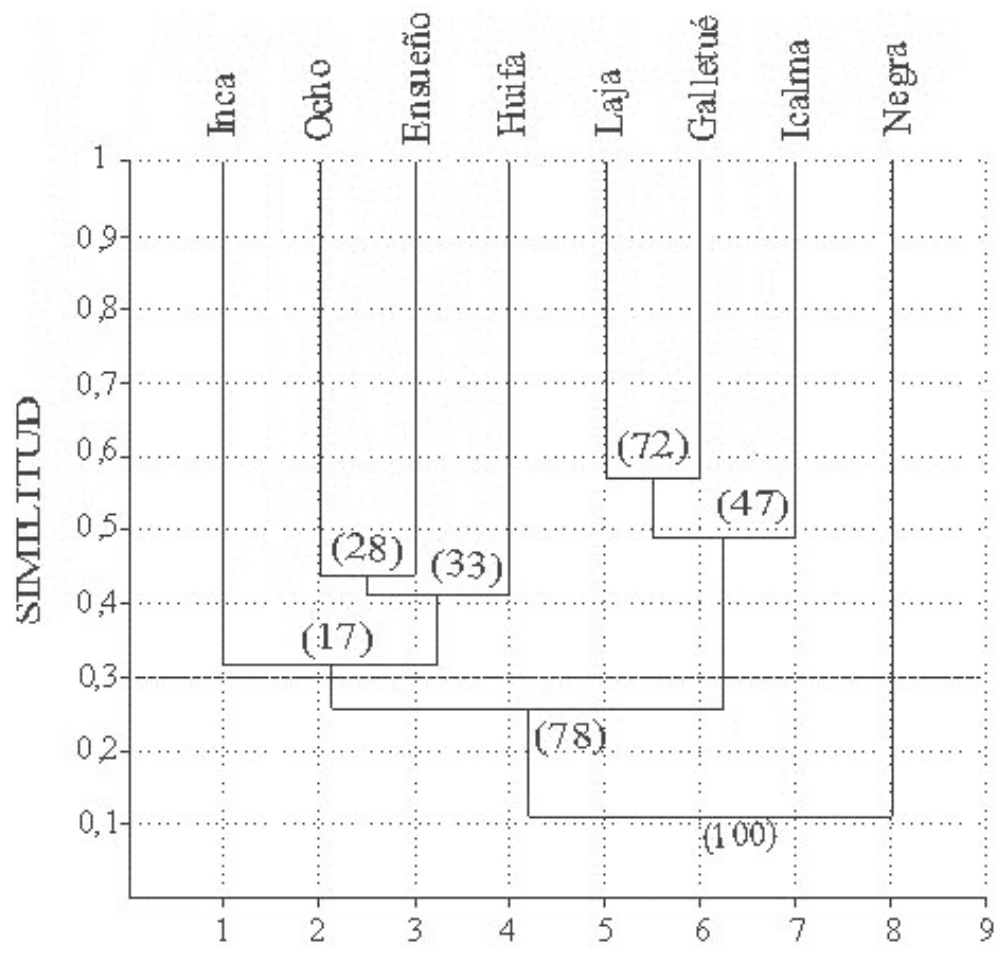

Fig. 2: Dendrogramas de los sitios muestreados utilizando el índice de Morisita y el ligamiento UPGMA. Entre paréntesis aparece el porcentaje de réplicas de "bootstrap" (100 permutaciones).

Cluster analysis of the sampling sites using Morisita index and UPGMA linkage. In parenthesis the proportion of similar replicated from bootstrapping (100 permutations) were indicated. 

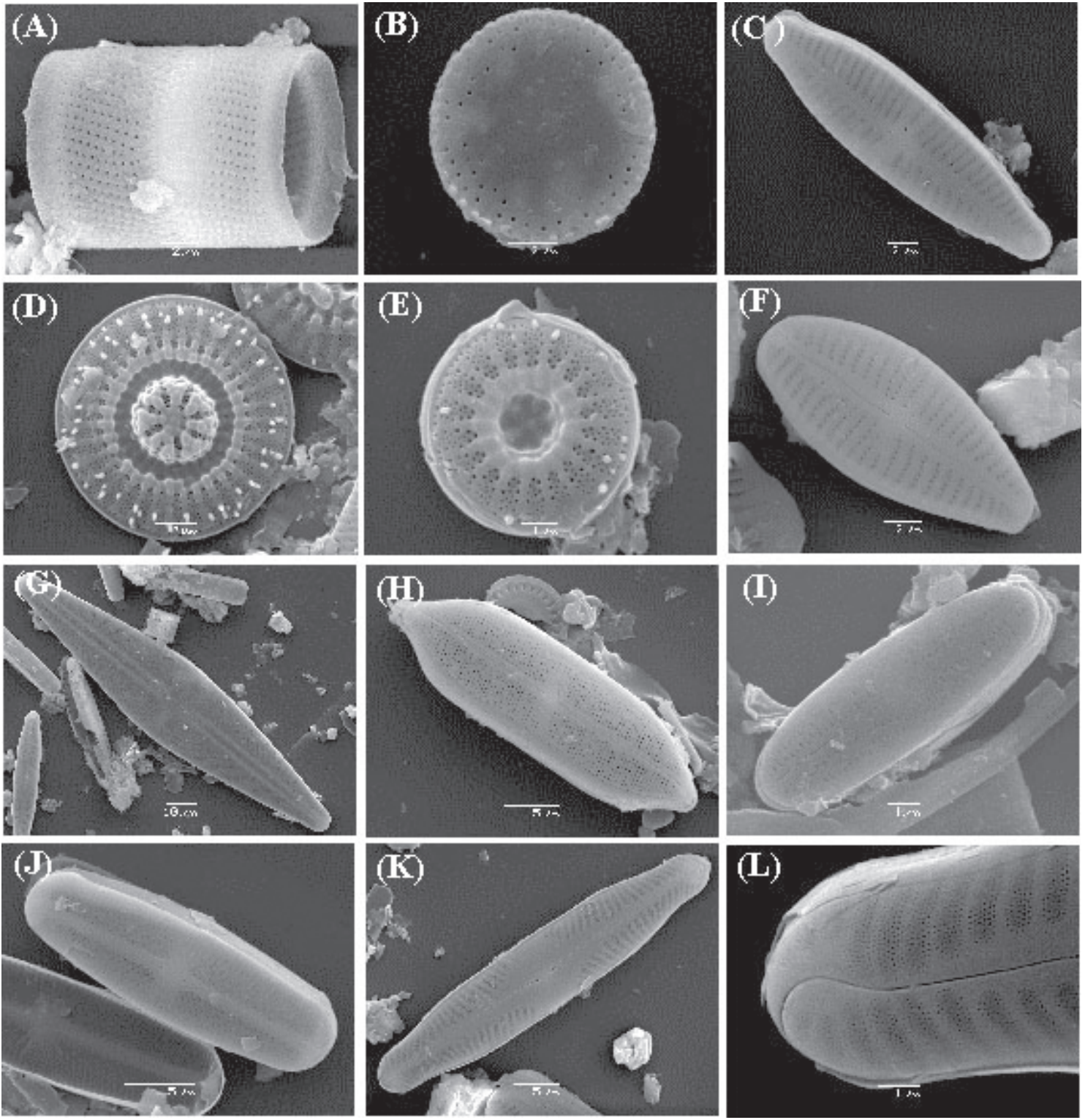

Fig. 3: Fotografías al microscopio electrónico de barrido de diatomeas encontradas en los sedimentos de los lagos andinos: (A) Aulacoseira alpigena, vista conectival, (B) A. alpigena, vista valvar, (C) Gomphonema araucana, vista externa, (D) Discostella stelligera, vista externa, (E) D. stelligera, vista interna, (F) Gomphonema acuminatum, vista externa, (G) Frustulia rhomboides, vista externa, (H) Neidium iridis, vista externa, (I) Especie sp.1, vista externa, (J) Achnanthes minutissimum, vista externa, (K) Pinnularia cf. acidicola, vista externa, (L) P. cf. acidicola, detalle del extremo del rafe.

Scanning Electron Microscopy (SEM) photographs of diatoms present in Andean Lakes: (A) Aulacoseira alpigena, conectival view, (B) A. alpigena, valve view, (C) Gomphonema araucana, external view, (D) Discostella stelligera, external view, (E) D. stelligera, internal view, (F) Gomphonema acuminatum, external view, (G) Frustulia rhomboides, external view, (H) Neidium iridis, external view, (I). Especie sp.1, external view, (J) Achnanthes minutissimum, external view, (K) Pinnularia cf. acidicola, external view, (L) P. cf. acidicola, detail of extreme of raphe. 

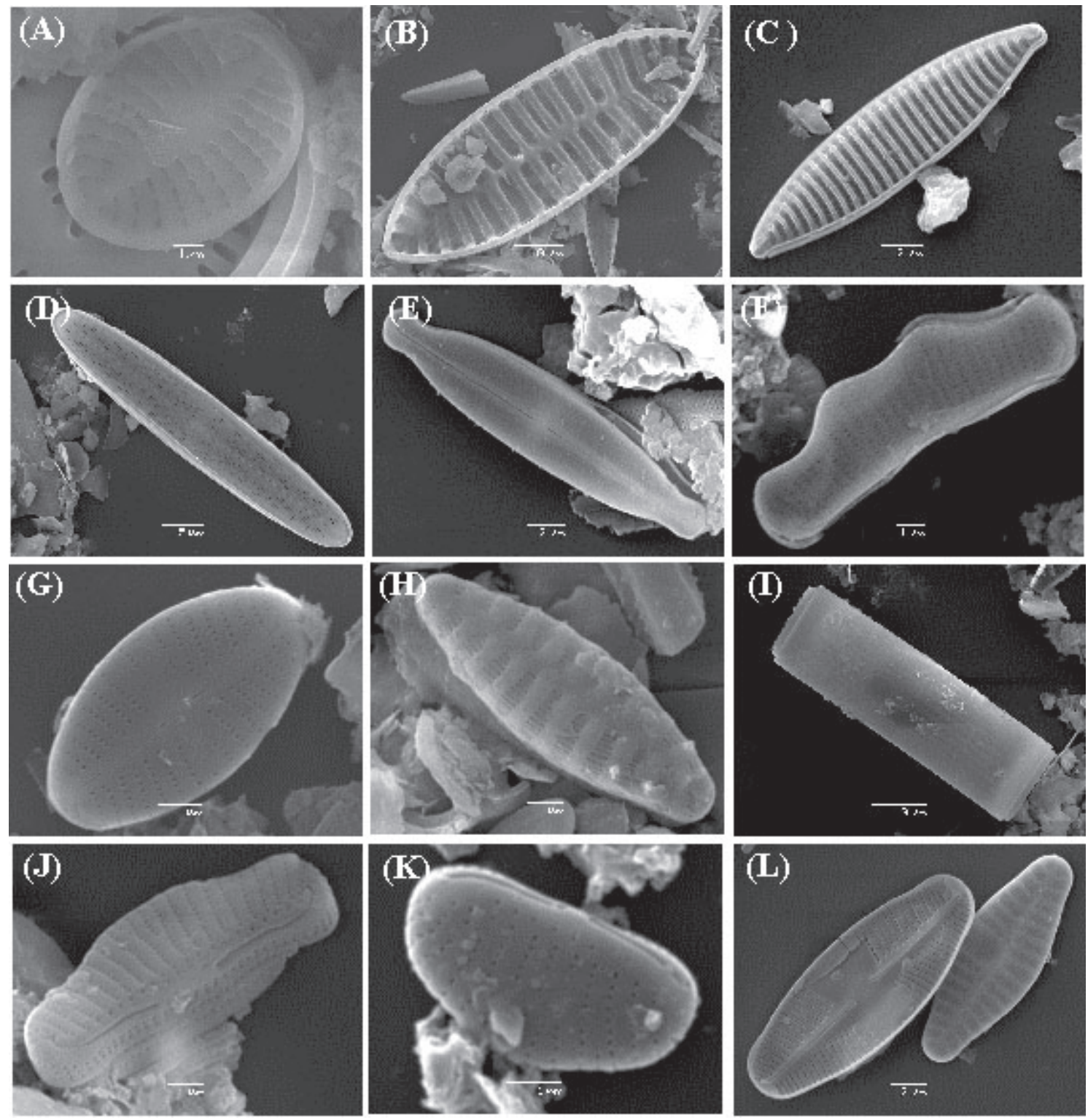

Fig. 4: Fotografías al microscopio electrónico de barrido de valvas de diatomeas encontradas en los sedimentos de los lagos andinos: (A) Especie sp.2, vista interna, (B) Surirella linearis, vista interna, (C) Staurosira sp., vista externa, (D) Caloneis sp., vista interna, (E) Kobayasiella sp., vista externa, (F) Eunotia diodon, vista externa, (G) Eolimna sp., vista externa, (H) Staurosirella sp., vista externa, (I) Melosira sp., vista conectival, (J) Eunotia sp.1, vista conectival, (K) Eunotia sp.2, vista externa y (L) Planothidium lanceolatum, vista externa e interna.

Scanning Electron Microscopy (SEM) photographs of diatoms present in Andean Lakes: (A) Especie sp.2, internal view (B) Surirella linearis, external view, (C) Staurosira sp., external view, (D) Caloneis sp., internal view, (E) Kobayasiella sp., external view, (F) Eunotia Diodon, external view, (G) Eolimna sp., external view, (H) Staurosirella sp., external view, (I) Melosira sp., conectival view, (J) Eunotia sp.1, conectival view, (K) Eunotia sp.2, external view y (L) Planothidium lanceolatum, internal and external view. 
los lagos ácidos muestran una predominancia de unos pocos taxa generalmente especies acido-tolerantes (Havens \& Carlson 1998). En el periodo de muestreo laguna Negra, presentó un valor de $\mathrm{pH}$ de 4,3. En esta área de los Andes no existen estudios geológicos, por lo tanto no es posible explicar los valores de $\mathrm{pH}$ observados. Sin embargo, al momento de la extracción y análisis de las muestras fue posible observar una gran cantidad de materiales arcillosos en los sedimentos de este sistema andino. Lo anterior podría ser resultado de la oxidación que presentan algunos materiales arcillosos como la pirita que puede dar lugar a la formación de ácido sulfúrico aportando con bajos valores de $\mathrm{pH}$ en este sistema (Whitton \& Díaz 1981). Esto podría responder a la peculiar composición diatomológica encontrada en laguna Negra.

A pesar de las diferencias observadas en la composición taxonómica de los distintos ensambles diatomológicos analizados, hubo varias especies presentes en ambos grupos de lagos, como es el caso de E. minutum, $P$. lanceolatum (Fig. 4L), D. stelligera (Fig. 3D y 3E) y A. minutissimum (Fig. 3J). En relación a lo anterior, Vila \& Muhlhauser (1987) mencionan que las asociaciones fitoplanctónicas de los lagos de altura se asemejan más a lagos templados y fríos andinos que a lagos tropicales de altura predominando en ellos especies cosmopolitas.

A su vez, Parra et al. (1993) señalan que el patrón de similitud observado en los lagos Icalma y Galletué parece estar relacionado con la historia geológica del área, presentando más similitudes a lagos araucanos precordilleranos que a lagos de altura. En efecto, varias de las especies que dominaron en estos sistemas entre ellas A. granulata, A. formosa, Hannaea arcus (Ehrenberg) Patrick y C. meneghiniana, especies cosmopolitas, planctónicas de ambientes más enriquecidos (Reynolds 2002) no fueron observadas en las lagunas de altura pero sí se encuentran señaladas en lagos araucanos y costeros del sur de Chile (Rivera et al. 1982, Cruces et al. 2001, Cruces et al. 2006).

Dado que la mayor cantidad de especies indeterminadas se encontraron en los lagos ubicados por sobre los $3.000 \mathrm{~m}$ de altura en la cordillera de los Andes, y que trabajos anteriores (Rumrich et al. 2000) señalan la posible existencia de especies endémicas para esta localidad, es de gran interés ecológico realizar posteriores estudios de la flora diatomológica en estos sistemas. De igual modo, se sugiere el estudio de las comunidades planctónicas para establecer comparaciones con las comunidades bentónicas que habitan en estos lagos. Por otro lado, los sistemas de altura estudiados se encuentran en estado prístino lo que se resalta su importancia como objetos de estudios de procesos naturales como estabilidad y clima.

\section{AGRADECIMIENTOS}

Esta investigación fue financiada por los proyectos FONDECYT N ${ }^{\circ} 1070508$ Y DIUC $\mathrm{N}^{\circ}$ 205.310.044-1.0 SP, Dirección de Investigación de la Universidad de Concepción.

\section{LITERATURA CITADA}

ANDERSON N \& R BATTARBEE (1994) Aquatic community persistence and variability: a paleolimnological perspective. En: Giller P, A Hildrew \& D Raffeli (eds) Aquatic ecology: scale, pattern and process: 233-259. Blackwell Scientific Publications, Oxford, United Kingdom.

BARBER H \& J CARTER (1996) An atlas of British diatoms. Biopress Limited, Bristol, United Kingdom. 600 pp.

BATTARBEE R (1986) Diatoms analysis. Handbook of Holocene palaeoecology and palaeohydrology. John Wiley \& Sons, New York, New York, USA. 570 pp.

BATTARBEE R (2000) Palaeolimnological approaches to climate change, with special regard to the biological record. Quaternary Science Reviews 19: 107-124.

BIGLER C \& R HALL (2002) Diatoms as indicators of climatic and limnological change in Swedish Lapland: a 100-lake calibration set and its validation for paleoecological reconstructions. Journal of Paleolimnology 27: 97-115.

BRUGAM R (1983) The relationship between fossil diatom assemblages and limnological conditions. Hydrobiologia 98: 223-235.

CATALÁN J, E BALLESTEROS, E GARCÍA, A PALAU \& L CAMARERO (1993) Chemical composition of disturbed and undisturbed high mountain lakes in the Pyrenees: a reference for acidified sites. Water Research 27: 133-141.

CHARLES D, J SMOL \& D ENGSTROM (1994) Paleolimnological approaches to biological M monitoring. En: Loeb LL \& A Spacie (eds) Biological monitoring of aquatic systems: 233-293. CRC Press, Florida, USA.

CHRISTIE C \& J SMOL (1993) Diatom assemblages as indicators of lake trophic status in southeastern Ontario lakes. Journal of Phycology 29: 575-586.

CONAF (2004) Antecedentes sobre las áreas silvestres protegidas de la Octava Región del Biobío. Editor Iván Benoit Contensee, Concepción, Chile. 28pp.

CRUCES F, R URRUTIA, A ARANEDA, L TORRES, M 
CISTERNAS Y W VYVERMAN (2001) Evolución trófica de Laguna Grande de San Pedro (VIII Región, Chile) durante el último siglo, mediante el análisis de registros sedimentarios. Revista Chilena de Historia Natural 74: 407-418.

CRUCES F, R URRUTIA, O PARRA, A ARANEDA, H TREUTLER, S BERTRAND, N FAGEL, L TORRES, R BARRA \& L CHIRINOS (2006) Changes in diatom assemblages in an Andean lake in response to a recent volcanic event. Archiv für Hydrobiologie 165: 23-35.

DENICOLA D (2000) A review of diatoms found in highly acidic environments. Hydrobiología 433: 111-122.

DÍAZ C \& N MAIDANA (2005) Diatomeas de los salares Atacama y Punta Negra, II Región, Chile. Centro de Ecología Aplicada Ltda., Santiago, Chile, 146 pp.

FLOWER R (1986) The relationship between surface sediment assemblages and $\mathrm{pH}$ in 33 Galloway lakes: some regression models for reconstructing $\mathrm{pH}$ and their application to sediment cores. Hydrobiología 143: 93-103.

GELL P (1998) Quantitative reconstruction of the Holocene palaeosalinity of paired crater lakes based on a diatom transfer function. Palaeoclimates 3: 83-96.

HAMMER O, D HARPER \& P RYAN (2001) PAST: paleontological statistics software for education and data analysis. Paleontología Electrónica 4: 1-9.

HASLE G \& G FRYXELL (1970) Diatoms: cleaning and mounting for light and electron microscopy. Transactions of the American Microscopical Society 89: 469-474.

HAVENS K \& R CARLSON (1998) Functional complementary in plankton communities along a gradient of acid stress. Environmental Pollution 101: 427-436.

KILROY C, B BIGGS, W VYVERMAN \& P BROADY (2006) Benthic diatoms communities in subalpine pools in New Zealand: relationships to environmental variables. Hydrobiologia 561: 95-110.

KRAMMER K \& H LANGE-BERTALOT (1991) Süsswasserflora von Mitteleuropa Bacillariophyceae Teil: Achnanthaceae. Fischer Verlag, Stuttgart, Germany. 437 pp.

KRAMMER K \& H LANGE-BERTALOT (2000) Süsswasserflora von Mitteleuropa Bacillariophyceae Teil: Centrales, Fragilariaceae, Eunotiaceae. Fischer Verlag, Stuttgart, Stuttgart, Germany. 598pp.

KREBS C (1989) Ecological methodology. Harper \& Row, New York, New York, USA. 652pp.

MANLY B (1997) Randomization, Boostrap and Montecarlo methods in biology. Chapman \& Hall, London, United Kingdom. 399 pp.

PARRA O, H CAMPOS, W STEFFEN, G AGÜERO, S BASUALTO, D AVILÉS \& M VIGHI (1993) Estudios limnológicos de los lagos Icalma y Galletué: lagos de origen del río Biobío (Chile Central). Serie monografías científicas del Centro EULA-Chile 12: 161-168.

RAUTIO M, S SORVARI \& A KORHOLA (2000) Diatom and crustacean zooplankton communities, their seasonal variability and representation in the sediments of subarctic Lake Saanajärvi. Journal of Limnology 59: 81-96.

REYNODLS C, S MORISON \& C BUTTERWICK (1982) the sedimentary flux of phytoplankton in the south basin of Windermere. Limnology and Oceanography 27: 1162-1175.

REYNOLDS C, V HUSZAR, C KRUK, L NASELLI \& S MELO (2002) Towards a functional classification of the freshwater phytoplankton. Journal of Plankton Research 24: 417-428.

RIVERA P (1970) Diatomeas de los lagos Ranco, Laja y Laguna Chica de San Pedro, Chile. Gayana Botánica (Chile) 20: 1-25.

RIVERA P, O PARRA, M GONZÁLES, V DELLAROSSA \& M ORELLANA (1982) Manual taxonómico del fitoplancton de aguas continentales. IV Bacilllariophyceae. Editorial Universidad de Concepción, Concepción, Chile. 126pp.

RIVERA R \& F CRUCES (2005) Stephanodiscus kuetzingii Klee \& Casper (Bacillariophyceae) from living material collected in Rapel Reservoir, Central Chile. Diatoms Research 20: 163-170.

RIVERA P (2006) Estado de conocimiento de las diatomeas dulceacuícolas de Chile. Gayana (Chile) 70: 1-7.

ROUND F, R CRAWFORD \& D MANN (1990) The diatoms: biology and morphology of the genera. Cambridge University Press, Cambridge, United Kingdom. 747pp.

RUMRICH U, H LANGE-BERTALOT \& M RUMRICH (2000) Diatomeen der Anden: Von Venezuela bis Patagonien/Feuerland und zwei weitere Beiträge. Iconographia Diatomológica, Volume 9. Koeltz scientific Books, Koenigstein, Germany. 673pp.

SMOL J \& B CUMMING (2000) Tracking long-term changes in climate using algal indicators in lake sediments. Journal of Phycology 36: 986-1011.

TAPIA P, E THERIOT, $S$ FRITZ, F CRUCES \& $P$ RIVERA (2004) Distribution and morphometric analysis of Cyclostephanos andinus comb. Nov., a planktonic diatom from the central Andes. Diatom Research 19: 311-327.

URRUTIA R, K SABBE, F CRUCES, K POZO, J BECERRA, A ARANEDA, W VYVERMAN \& O PARRA (2000) Paleolimnological studies of Laguna Chica of San Pedro (VIII Región): diatoms, hydrocarbons and fatty acid records. Revista Chilena de Historia Natural 73: 717-728.

VAN DAM H, A MERTENS \& J SINKELOAM (1994) A coded checklist and ecological indicator values of freshwater diatoms from the Netherlands. Netherlands Journal of Aquatic Ecology 28: 117-133.

VAN DE VIJVER B, L BEYENS, S VINCKE \& $N$ GREMMEN (2004) Moss-inhabiting diatom communities from Heard Island, sub-Antarctic. Polar Biology 27: 532-543.

VILA I \& H MÜHLHAUSER (1987) Dinámica de lagos de altura, perspectivas de investigación. Archivos de Biología y Medicina Experimentales (Chile) 20: $95-$ 103.

WHITTON B \& B DÍAZ (1981) Influence of environmental factors on photosynthetic species composition in highly acidic waters. Internationale Vereinigung für Theoretische und Angewandte Limnologie 21: 1459-1465.

WOLDA H (1981) Similarity indices, samples size and diversity. Oecología 50: 296-302.

WUNSAM S, R SCHMIDT \& R KLEE (1995) Cyclotella taxa (Bacillariophyceae) in lakes of the Alpine region and their relationship to environmental variables. Aquatic Sciences 57: 360-386. 